\title{
Bleeding diathesis and gastro-duodenal ulcers in inherited cytosolic phospholipase-A2 alpha deficiency
}

\author{
Elena M. Faioni'; Cristina Razzari'; Aida Zulueta2; Eti A. Femia'; Lara Fenu³; Marco Trinchera4; Gian Marco Podda'; \\ Mariateresa Pugliano ${ }^{1}$; Francesco Marongiu ${ }^{3}$; Marco Cattaneo ${ }^{1}$ \\ ${ }^{1}$ Medicina 3, Azienda Ospedaliera San Paolo and Dipartimento di Scienze della Salute, Università degli Studi di Milano, Milano, Italy; ${ }^{2}$ Dipartimento di Scienze della Salute, \\ Università degli Studi di Milano, Milano, Italy; ${ }^{3}$ Dipartimento di Scienze Mediche Internistiche, Università di Cagliari, Cagliari, Italy; ${ }^{4}$ Dipartimento di Medicina Clinica e \\ Sperimentale, Università dell'Insubria, Varese; Italy
}

\begin{abstract}
Summary
Arachidonic acid (AA), when cleaved from phospholipids by cytosolic phospholipase A2 alpha (CPLA2a), generates eicosanoids, with pro-hemostatic, pro-inflammatory, vasoactive and gastro-protective functions. We describe a patient (27-year-old man) and his twin-sister with early-onset bleeding diathesis and recurrent gastro-intestinal (GI) ulcers. Platelet aggregation/ $\delta$-granules secretion by collagen was impaired, but normal by $A A$; serum levels of thromboxane $(T x) B_{2}$ and 12-hydroxyeicosatetraenoic acid, and urinary levels of 11-dehydro$\mathrm{TxB}_{2}$ were extremely low. Patients were homozygous for $1723 \mathrm{G}>\mathrm{C}$ transition in PLA2G4A gene, which changed the codon for Asp575 to His. GI ulcers affected $5 / 14$ heterozygous ( $<40$ years) and $1 / 16$ wildtype homozygous ( $>60$ years) family members; none had bleeding diathesis. The proband, his sister and mother also had mildly reduced
\end{abstract}

\section{Correspondence to:}

Marco Cattaneo, MD

Medicina 3, Ospedale San Paolo

Dipartimento di Scienze della Salute

Università degli Studi di Milano

Via A. di Rudinì, 8

20142 Milano, Italy

Tel.: +39 02 50323188, Fax: +390250323089

E-mail: marco.cattaneo@unimi.it factor XI levels. Platelet messenger RNA expression did not differ among subjects with different PLA2G4A genotypes. Conversely, platelet CPLA2a was undetectable by Western Blotting in the proband and his sister, and decreased in $1723 \mathrm{G}>C$ heterozygous subjects, suggesting that the variant is transcribed, but not translated or translated into an unstable protein. We described a syndromic form of deficiency of CPLA2a , characterised by recurrent GI ulcers and bleeding diathesis, associated with mild inherited deficiency of factor XI. Unlike other reported patients with CPLA2a deficiency, these patients had extremely low levels of platelet $\mathrm{TXA}_{2}$ biosynthesis.

\section{Keywords}

Platelet function disorders, bleeding diathesis, phospholipase A2a deficiency, eicosanoids, gastro-duodenal ulcers

\section{Received: April 14, 2014}

Accepted after major revision: June 20, 2014

Epub ahead of print: August 7, 2014

http://dx.doi.org/10.1160/TH14-04-0352

Thromb Haemost 2014; 112: $\mathrm{an}$

\section{Introduction}

Eicosanoids (prostaglandins, thromboxanes, leukotrienes, epoxyeicosatrienoic acids and lipoxins), are signalling molecules that are generated by the conversion of fatty acids, primarily arachidonic acid (AA), by cyclooxygenases, lipoxygenases and cytochrome P450-dependent epoxygenase (1). Cleavage of AA from membrane phospholipids by cytosolic phospholipase A2alpha (cPLA2a), is the rate limiting step in eicosanoid biosynthesis in many cells, notably those that undergo calcium dependent activation of the enzyme $(1,2)$. cPLA2a is a 749 amino acid long enzyme that has an $\mathrm{N}$-terminal calcium-binding domain, responsible for the interaction with membrane lipids, and a C-terminal catalytic domain, linked by a short and flexible peptide (2). The conserved active site is composed of a serine (Ser228) and an aspartic acid (Asp549) (3). The cPLA2a gene resides at chromosome 1q25 (PLA2G4A, Gene ID in HGNC:9035).

In platelets, $\mathrm{AA}$ is the precursor of prostaglandin endoperoxides and thromboxane (Tx) $\mathrm{A}_{2}$, potent platelet activating agents (4); congenital and drug-induced inhibition of their synthesis is associated with mildly increased risk of bleeding $(5,6)$. Through binding to G-protein-coupled receptors, eicosanoids mediate inflammation and promote release of mitogenic and angiogenic factors (1). Some AA-derived prostaglandins, such as prostaglandins $\mathrm{E}_{2}, \mathrm{I}_{2}$ and $\mathrm{F}_{2} \alpha$, maintain gastric mucosal integrity by inhibiting acid secretion, stimulating mucus and bicarbonate secretion, inhibiting mast cell activation, decreasing leukocyte adherence to the vascular endothelium, inhibiting apoptosis, increasing or maintaining mucosal blood flow and thus preventing ischaemia (7). It was shown that inhibition of prostaglandins by nonsteroidal anti-inflammatory drugs (NSAIDS) or antibodies leads to increased acid secretion, reduced proliferative potential and angiogenesis, increased susceptibility to injury by acid and toxic substances, and ulcer formation $(8,9)$. 
Considering the pleiotropic actions of AA derivatives, a deficiency in its metabolic pathway is expected to be associated with syndromic disease in man. In this report, we describe a syndromic form of inherited deficiency of cPLA2a, characterised by GI ulcers and a bleeding diathesis associated with platelet dysfunction, deriving from the severe impairment of AA metabolism, and associated with mild factor XI deficiency.

\section{Methods}

\section{Materials}

Adenosine diphosphate (ADP), the thromboxane/prostaglandin endoperoxide analogue 9,11-dideoxy-11,9-epoxymethano-prostaglandin $F_{2}$ (U46619), thrombin receptor activating peptide (TRAP; Ser-Phe-Leu-Leu-Arg-Asn-Pro-Asn-Asp-Lys-Tyr-GluPro-Phe) were from Sigma Aldrich (St. Louis, MO, USA). Horm collagen was from Mascia Brunelli (Milan, Italy). Commercial preparations of luciferin/luciferase reagent were used to measure the platelet adenosine triphosphate (ATP) and ADP contents (ATP Assay Kit, Promega Italia, Milan, Italy), and platelet ATP released concurrently with platelet aggregation (Chronolume, Chrono-log Corp, Havertown, PA, USA).

\section{The Proband}

The proband is a 27-year-old Caucasian man, born in Sardinia, Italy. He has a lifelong bleeding diathesis with repeated spontaneous mucous bleeding events (epistaxis, gum bleeding, GI bleeding, haematuria) and post-surgery haemorrhage. His bleeding score, which was calculated using the ISTH/SSC bleeding assessment tool $(10)$ is $9($ normal $\leq 3)$ ( $>$ Table 1$)$. He suffers from recur- rent duodenal ulcers, starting at the age of 24 months, which were diagnosed by endoscopy. He was found Helicobacter pylori positive, and the infection was eradicated. Since gastro-duodenal bleeding occurred though he had been taking inhibitors of the gastric proton pump, the synthetic prostaglandin $\mathrm{E}_{1}$ misoprostol was administered for the last two years and he has not experienced further gastrointestinal (GI) bleeding. Because this intervention corrected the GI problems, he has not had small bowel endoscopy, and the presence or absence of small bowel ulcers in unknown. He currently has a mildly reduced renal function (creatinine clearance: $50 \mathrm{ml} /$ minute [min]), which was observed at age 13 years for the first time, and has since stabilised.

\section{The twin sister of the proband}

The twin sister has a personal history similar to the proband. She also has a lifelong bleeding diathesis, with spontaneous muco-cutaneous bleeding (gum bleeding, spontaneous haematomas $>1 \mathrm{~cm}$, GI bleeding, haematuria and severe menorrhagia), which caused the development of iron deficiency anaemia. Her bleeding score was 11. Like her twin brother, she suffered from duodenal ulcers (diagnosed by endoscopy), starting at the age of 24 months, was treated with proton pump inhibitors and subsequently with the addition of misoprostol.

\section{Other family members}

Family members of the proband make up a large kindred, living in different parts of Italy. Personal and family history, blood samples, copies of pertinent medical documentation, including results of recent blood tests and esophagogastroduodenoscopy, were collected and the bleeding score was calculated for each member.

Table 1. Relationship between $\mathrm{CPLA}_{2}$ a genotypes and clinical and laboratory phenotypes

\begin{tabular}{|c|c|c|c|c|c|}
\hline & \multirow{3}{*}{$\begin{array}{l}\text { Healthy } \\
\text { controls } \\
n=25\end{array}$} & \multicolumn{4}{|c|}{ Family members } \\
\hline & & \multirow{2}{*}{$\begin{array}{l}\text { Wild type } \\
n=21\end{array}$} & \multirow{2}{*}{$\begin{array}{l}\text { Heterozygous Asp575His } \\
n=14\end{array}$} & \multicolumn{2}{|c|}{ Homozygous Asp575His } \\
\hline & & & & proband & twin sister \\
\hline $\begin{array}{l}\text { Serum } \mathrm{TXB}_{2}, \mathrm{ng} / \mathrm{ml} \\
\text { [median (range)] }\end{array}$ & $\begin{array}{l}175 \\
(41-608)\end{array}$ & $\begin{array}{l}164 \\
(159-321)\end{array}$ & $\begin{array}{l}187 \\
(54-420)\end{array}$ & 0.17 & 0.11 \\
\hline $\begin{array}{l}\text { Urinary 11-dehydro TXB2, } \\
\text { pg/mg creatinine } \\
\text { [median (range)] }\end{array}$ & $\begin{array}{l}364 \\
(148-658)\end{array}$ & $\begin{array}{l}341 \S \\
(208-314)\end{array}$ & $\begin{array}{l}304^{*} \\
(163-644)\end{array}$ & 96 & 67 \\
\hline Serum 12-HETE, pg/ $\mu$ l [median (range)] & - & $\begin{array}{l}319^{\S} \\
(242-775)\end{array}$ & $\begin{array}{l}213^{*} \\
(114-575)\end{array}$ & 25 & 15 \\
\hline Bleeding score & $\leq 3$ & $1-4$ & $1-3$ & 9 & 11 \\
\hline Documented gastric or duodenal ulcer & - & $2 / 21(10 \%)$ & $5 / 14(36 \%)$ & yes & yes \\
\hline Age at first gastric or duodenal ulcer, years & - & $>60$ & $<40$ & 2 & 2 \\
\hline Chronic gastritis & - & $2 / 21(10 \%)$ & $3 / 14(21 \%)$ & no & no \\
\hline Helicobacter pylori infection & - & $0 / 21(0 \%)$ & $5 / 14(36 \%)$ & yes & no \\
\hline
\end{tabular}


All subjects were informed of the purpose of the study and an informed consent was signed by every subject included in the present report.

\section{Blood sampling}

Blood from the proband and from the family members was drawn in trisodium citrate $(109 \mathrm{mmol} / \mathrm{l}, 9$ volumes of blood:1 volume of anticoagulant) for coagulation, von Willebrand Factor (VWF) measurement and platelet function studies, in $\mathrm{K}$ - ethylenediaminetetraacetic acid (EDTA) for DNA/RNA extraction and blood cell counts, in acid-citrate-dextrose (ACD, 6 volume of blood:1 volume of anticoagulant) to prepare washed platelet suspensions, and in no anticoagulant to measure serum $\mathrm{TxB}_{2}$, which is a stable metabolite of $\mathrm{TxA}_{2}$, and 12-hydroxyeicosatetraenoic acid (HETE).

\section{Urine sampling}

A $10-\mathrm{ml}$ of overnight urine sample was collected, stored at $-80^{\circ} \mathrm{C}$ until measurement of 11-dehydro-thromboxane $\mathrm{B}_{2}$ and creatinine

\section{Platelet aggregation and secretion studies}

Platelet aggregation and ATP secretion induced by ADP ( 2 and 4 $\mu \mathrm{mol} / \mathrm{l})$, collagen $(2$ and $4 \mu \mathrm{g} / \mathrm{ml})$, AA ( $1 \mathrm{mmol} / \mathrm{l})$, (TRAP) (10 $\mu \mathrm{mol} / \mathrm{l})$, epinephrine $(5 \mu \mathrm{mol} / \mathrm{l})$, and the thromboxane analogue $\mathrm{U} 46619(1 \mu \mathrm{mol} / \mathrm{l})$ in platelet-rich plasma (PRP) were measured by lumiaggregometry (Chronolog Corporation) (11).

PRP was prepared by centrifugation of citrate-anticoagulated blood samples at $200 \times g$, at room temperature (RT) for $10 \mathrm{~min}$ (12); platelet-poor plasma (PPP) was prepared by centrifugation of blood samples at $1400 \times g$ at RT for $15 \mathrm{~min}$. The platelet count in PRP was not adjusted to a standardised value, because this practice may alter platelet function $(13,14)$.

\section{Measurement of other parameters of haemostasis}

Prothrombin time and activated partial thromboplastin time were measured by standard methods. VWF activity was measured by latex particle-enhanced immunoturbidometric assay (Instrumentation Laboratory, Milan, Italy). PFA'-100 (Siemens, Milan, Italy) closure times were evaluated using both the collagen-ADP and the collagen-epinephrine cartridges.

\section{Eicosanoid measurements}

Serum samples were prepared from whole blood that had been allowed to clot at $37^{\circ} \mathrm{C}$ for 1 hour, and frozen at $-20^{\circ} \mathrm{C}$ until assay by a commercially available ELISA for $\mathrm{TxB}_{2}$ (Cayman Chemical Company, Ann Arbor, MI, USA), and by liquid chromatography/ tandem mass spectrometry for 12-HETE (15). Urinary levels of 11-dehydro- $\mathrm{TXB}_{2}$ were measured using a previously described radioimmunoassay technique validated by comparison with gas chromatography - mass spectrometry (GC-MS) (16); data were corrected for the urinary levels of creatinine.

\section{CPLA2a genomic DNA sequencing}

The 18 exons of the PLA2G4A gene were amplified from genomic DNA, using sequence-specific primers. Polymerase Chain Reactions (PCRs) were performed with DreamTaq Green Polymerase, 10x DreamTaq Green (Fermentas ThermoFisher Scientific Inc, Waltham, MA, USA) and dNTP mix (Invitrogen-Life Technologies corp, Carlsbad, CA, USA). PCR products were sequenced by ABI 3730xl 96-capillary DNA Analysers (Eurofins MWG Operon, Huntsville, AL, USA).

\section{Amplification refractory mutation system (ARMS)}

Exon 15 of PLA2G4A gene was amplified from genomic DNA, using ARMS-specific primers. PCRs were performed with DreamTaq Green Polymerase (Fermentas), 10× DreamTaq Green (Fermentas), and dNTP mix (Invitrogen). To provide a suitable control, two PCR reactions were run in parallel, one with a primer matching the variant at the 3 ' end, and the other with the primer matching the parent sequence. The primer sequences were:

- Primer sense: 5'-AAGCCTGAGGGCCTAATCAT-3'

- Primer reverse : 5'-CTTGGCCTTGCAGAAAAGTC-3'

- Primer reverse with variant at the 3' end: 5'-CTTGGCCTTGCAGAAAAGTG-3'

\section{Competitive Reverse Transcription (RT)-PCR}

Quantification of cPLA2a transcript was performed by competitive RT-PCR, which allows to analyse long segments of the coding mRNA sequence, as reported (17). Briefly, cDNAs, synthesised as reported (18), were amplified in a volume of $25 \mu \mathrm{l}$ in the presence of the indicated amounts of competitor for 35 cycles (blood cPLA2a), or $5 \mathrm{pg}$ competitor for 25 cycles ( $\beta$-actin), using $2.5 \mathrm{U} / \mathrm{ml}$ of GoTaq Hot Start polymerase (Promega Italia, Milan, Italy) in a mixture containing the supplied buffer, $2.0 \mathrm{mM} \mathrm{MgCl} 2,0.1 \mathrm{mM}$ dNTPs, $250 \mathrm{pmol} / \mathrm{ml}$ of each primer pair (cPLA2a sense: 5'-GTGGAGCACCAGTATTCCCAC; CPLA $_{2} \mathrm{a}$ reverse: 5'-CAGGTTTGACATGAAGACAGGTG-3'), and different amounts of cDNAs. Program for cPLA2a amplification included a single treatment at $94^{\circ} \mathrm{C}$ for $3 \mathrm{~min}$ followed by cycles consisting of $1 \mathrm{~min}$ at $94^{\circ} \mathrm{C}$ (melting), $1 \mathrm{~min}$ at $62^{\circ} \mathrm{C}$ (annealing), and $1.5 \mathrm{~min}$ at $72^{\circ} \mathrm{C}$ (extension), and a final extension step at $72^{\circ} \mathrm{C}$ for $8 \mathrm{~min}$. Amplification program and competitor cDNA for $\beta$-actin were as reported (17). Competitor for CPLA2a was prepared with the same approach but using EcoRV and Eco72I for truncating the plasmid. Parallel PCR amplifications were performed on known amounts of standard cDNAs premixed with the competitors.

Aliquots of PCR fragments were analysed on $1 \%$ agarose gels stained with ethidium bromide. Quantification was performed by densitometric scanning of the gel and the amounts of amplified target cDNAs were calculated from their respective standard 
curves and normalised by those for $\beta$-actin. The target/competitor ratios were proved to make PCR results quantitative (19).

\section{Western blotting}

Platelets from ACD-PRP were washed four times in Tyrode's buffered solution containing $0.1 \mathrm{~g}$ glucose, $0.35 \%$ albumin from bovine serum, $5 \mathrm{mmol} / \mathrm{l} \mathrm{EDTA}, 1 \mu \mathrm{mol} / 1 \mathrm{PGE}_{1}(\mathrm{pH}=7.4)$, counted, diluted to $1 \times 10^{6} / \mu \mathrm{l}$, and incubated with lysis buffer $(50 \mathrm{mmol} / \mathrm{l}$ TRIS-HCl pH 7.4, $250 \mathrm{mmol} / \mathrm{l} \mathrm{NaCl}, 1 \%$ Triton X-100, $5 \mathrm{mmol} / \mathrm{l} \beta$ glycerol-phosphate, $1 \mathrm{mmol} / 1 \mathrm{Na}_{3} \mathrm{VO}_{4}, 15 \mathrm{mmol} / \mathrm{l} \mathrm{NaF}, 1 \mathrm{mmol} / \mathrm{l}$ phenylmethylsulfonyl fluoride), $2 \%$ protease inhibitor cocktail (Complete EDTA-free, Roche Diagnostics, Basel, Switzerland) and $0.5 \%$ protease inhibitor cocktail (Sigma-Aldrich) for $40 \mathrm{~min}$. The platelet lysate was frozen at $-20^{\circ} \mathrm{C}$ for $20 \mathrm{~min}$, thawed at RT, centrifuged $\left(16,000 \times g\right.$ at $4^{\circ} \mathrm{C}$ for $\left.15 \mathrm{~min}\right)$ and the supernatant recovered. Samples ( $34 \mu \mathrm{g}$ of protein) were separated by $4-12 \%$ TrisGlycine Gel SDS PAGE (KomaBiotech, Gangseo-gu Seoul, Korea), transferred to a nitrocellulose membrane with a semi-dry method (iBlot gel Tranfer System, Invitrogen-Life Technologies Corp.) and blotted with rabbit polyclonal anti- cPLA2a (sc-438, Santa Cruz Biotechnology Inc, Santa Cruz, CA, USA, 1:100) or rabbit poly- clonal anti- $\beta$-actin (Cell Signalling Technology Inc, Beverly, MA, USA, 1:1,000) as reported (18).

\section{Results}

\section{The Proband}

The proband had normal blood cells counts and prothrombin time; his activated partial thromboplastin time was slightly prolonged (24 seconds [s], ratio $=1.32$, normal range $0.8-1.2$ ), likely due to partial deficiency of factor XI ( $64 \%$, normal values $\geq 70 \%)$. His bleeding time was in excess of $20 \mathrm{~min}$ (normal values $<8 \mathrm{~min}$ ), and was shortened to $8.5 \mathrm{~min}$ by intravenous desmopressin $(0.3 \mu \mathrm{g} / \mathrm{kg})(20)$. The closure time of the collagen-ADP cartridge of PFA -100 was slightly prolonged (119 s, normal range 60-116), while that of the collagen-epinephrine cartridge was severely prolonged (>300 s, normal range 76-143). The above observations suggested that the patient had a defect of primary haemostasis despite normal platelet count, which is likely due to a platelet function abnormality, rather than von Willebrand disease (21). Indeed, the plasma levels of von Willebrand factor antigen and ristocetin cofactor activity were normal: therefore, we focused our attention on platelet function.

\section{A - Collagen $2 \mu \mathrm{g} / \mathrm{mL}$}

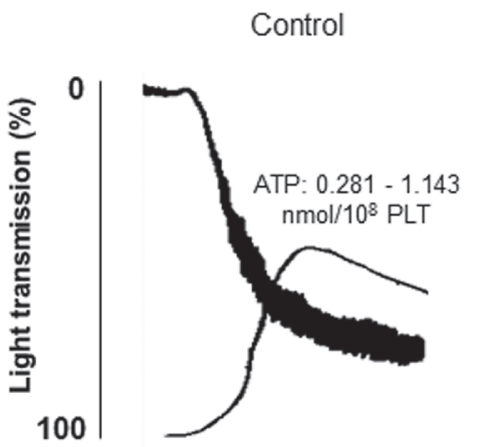

III-8

III-9
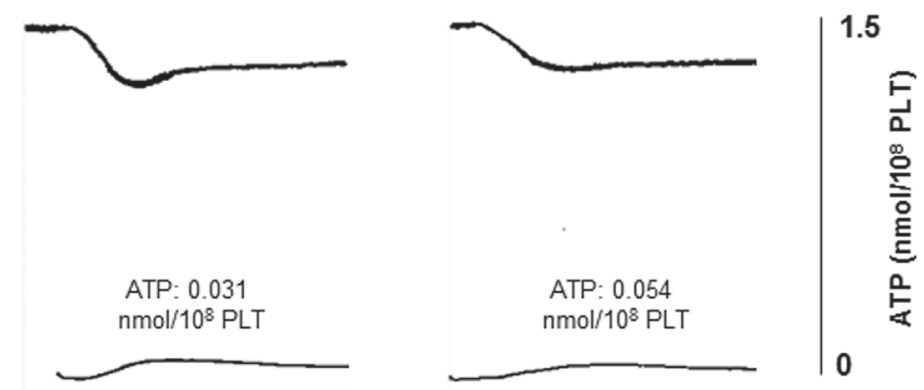

B - Arachidonic acid $1 \mathrm{mM}$

Control

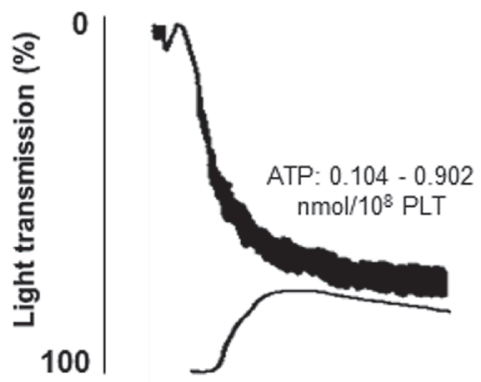

|| $1-8$

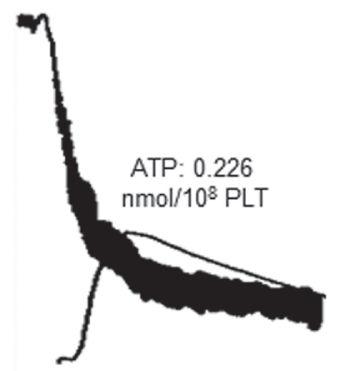

III-9

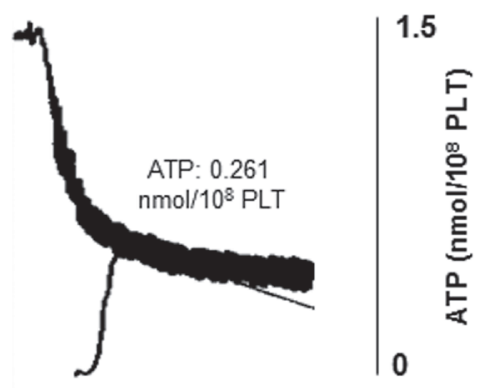

Figure 1: Platelet aggregation and ATP secretion of citrated PRP from the proband, his twin-sister, and healthy volunteers. Platelets were stimulated with collagen $2 \mu \mathrm{g} / \mathrm{ml}$ (A), or arachidonic acid $1 \mathrm{mM}(\mathrm{B})$. The extent of ATP secretion is reported close to the secretion tracings. 


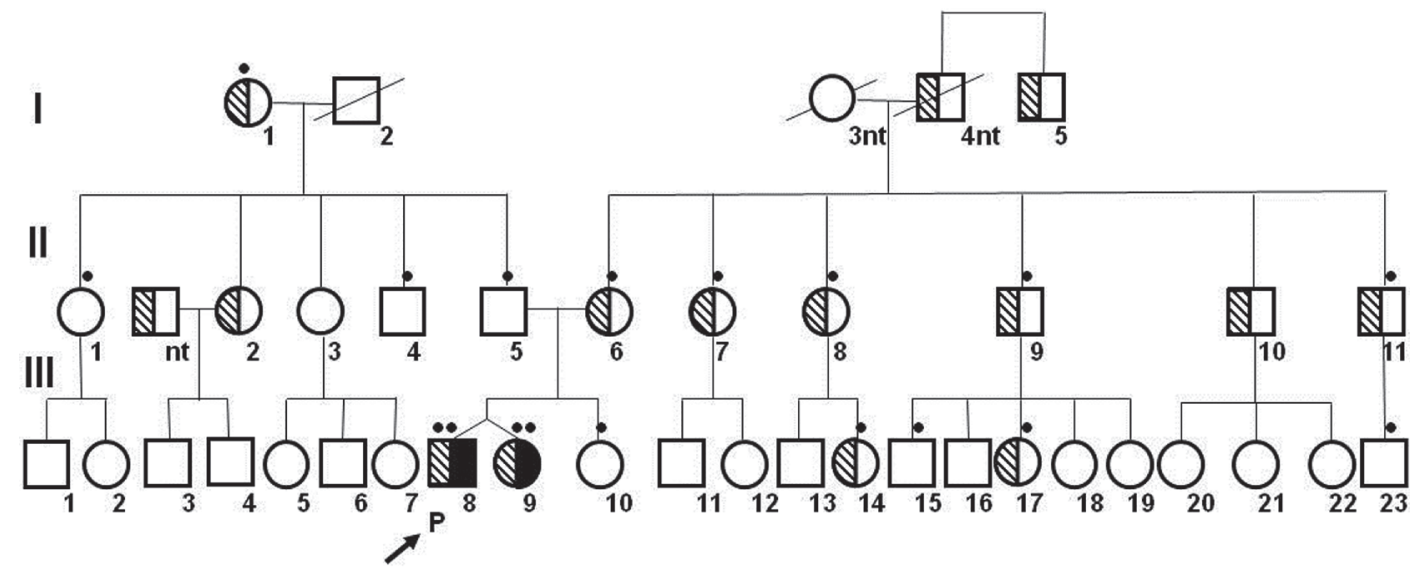

Figure 2: Family tree of the proband. Three generations are reported. Double dots= homozygous and single dot= heterozygous for the Asp575His variant. Black: bleeding diathesis; stripes= duodenal ulcer or chronic gastritis. The arrow designates the proband (P); the diagonal line across the symbol indicates deceased; $\mathrm{nt}=$ not genotyped.

\section{Platelet function studies}

Platelet aggregation and secretion induced by collagen were severely defective, while those induced by AA were normal $(\nabla$ Figure 1). Platelet aggregation and secretion induced by ADP, epinephrine, TRAP, or the $\mathrm{TxA}_{2}$ mimetic U46619 were defective (not shown). The platelet content of ADP and ATP was normal (not shown).

\section{Measurements of eicosanoids in serum and urine}

Serum $\mathrm{TxB}_{2}$ levels were extremely low ( Table 1 ), about three orders of magnitude lower than the median levels measured in healthy subjects, and 1 order of magnitude lower than those measured in healthy subjects who had been treated with $100 \mathrm{mg}$ aspirin daily for four days (median $2.99 \mathrm{ng} / \mathrm{ml}$, range $0.37-15.35$ $\mathrm{ng} / \mathrm{ml}$ in 29 individuals:) or in patients with coronary artery disease under chronic treatment with aspirin $100 \mathrm{mg}$ daily (median $2.50 \mathrm{ng} / \mathrm{ml}$, range $0.27-57.83 \mathrm{ng} / \mathrm{ml}$ in 49 patients) who were studied in our centre. The urinary levels of 11 -dehydro- $\mathrm{TxB}_{2}$ were also low ( Table 1$)$.

\section{The twin sister of the proband}

The blood cells count of the sister of the proband was normal; in contrast with her brother, both her PT and APTT, which were tested on two separate occasions both in Milan and in Cagliari, were absolutely normal: however, also her plasma factor XI levels were slightly decreased (62\%). The plasma levels of VWF were normal (not shown). The results of platelet function studies were superimposable to those of the proband ( $\nabla$ Figure 1 and $>$ Table $1)$.

Overall, the results of platelet function studies and eicosanoid measurement in the proband and his twin sister are consistent with a defect in the arachidonate pathway of platelet activation, with severely defective production of $\mathrm{TxA}_{2}$. Because AA-induced platelet functions were normal, we hypothesised that the proband and his twin sister had a defect of cPLA2a, which is responsible for cleavage of arachidonate from membrane phospholipids upon platelet activation and, consequently, for its metabolic transformation to active eicosanoids. The finding of extremely low serum levels of 12-HETE in both subjects ( Table 1), which is an arachidonate metabolite of the lypoxygenase pathway, was consistent with our hypothesis. Therefore, we looked for abnormalities of PLA2G4A, the gene encoding for cPLA2a.

\section{DNA sequencing of PLA2G4A}

The coding region of PLA2G4A was amplified and sequenced. The variant $1723 \mathrm{G}>\mathrm{C}$ was identified in homozygosity in the proband and the twin sister (BankIt1613963 55051.8.cPLA15.ex15S KC888000). The $1723 \mathrm{G}>\mathrm{C}$ variant was not found in 100 normal individuals by ARMS. Among the additional 35 family members studied, 21 (60\%) were homozygous for wild-type genotype, and $14(40 \%)$ were heterozygous for the $1723 \mathrm{G}>\mathrm{C}$ variant. The predicted amino acid change associated with the $1723 \mathrm{G}>\mathrm{C}$ variant is Asp575His, located in the catalytic domain of cPLA2a, which causes changes both in charge and bulk. Asp575 is highly conserved both trans-species and in the other isoenzymes of cytosolic phospholipases that belong to group 4, which show elevated homology to cPLA2a (22).

\section{Clinical and laboratory features of family members}

-Figure 2 shows the distribution of personal history of abnormal bleeding and ulcer or chronic gastritis in the family members of the proband and his twin sister who were available for study. Only GI ulcers that were documented by esophagogastroduodenoscopy images verified by the authors are reported. Heterozygotes for the cPLA2a variant did not have a bleeding diathesis, but had a 
relatively high prevalence of duodenal ulcers at young age $(<40$ years) ( Table 1). Relatives with wild-type cPLA2a genotype did not have a bleeding diathesis, and, compared to heterozygotes, had a lower prevalence of duodenal ulcers, which developed when they were older than 60 years of age ( Table 1). The levels of serum $\mathrm{TxB}_{2}$ and 12-HETE, and of urinary 11-dehydro- $\mathrm{TxB}_{2}$ were borderline-low or normal in heterozygotes, and normal in relatives with wild type genotype ( $\triangleright$ Table 1 ). The mother of the proband (II-6) had decreased plasma factor XI levels (42\%), but she never had pathological bleeding even on occasions of ingestions of aspirin as antipyretic and analgesic drug.

\section{Expression of cPLA2a variant in patients' platelets}

The amounts of cPLA2a transcript detected in patients' platelets were variable among individual members of the family, with no

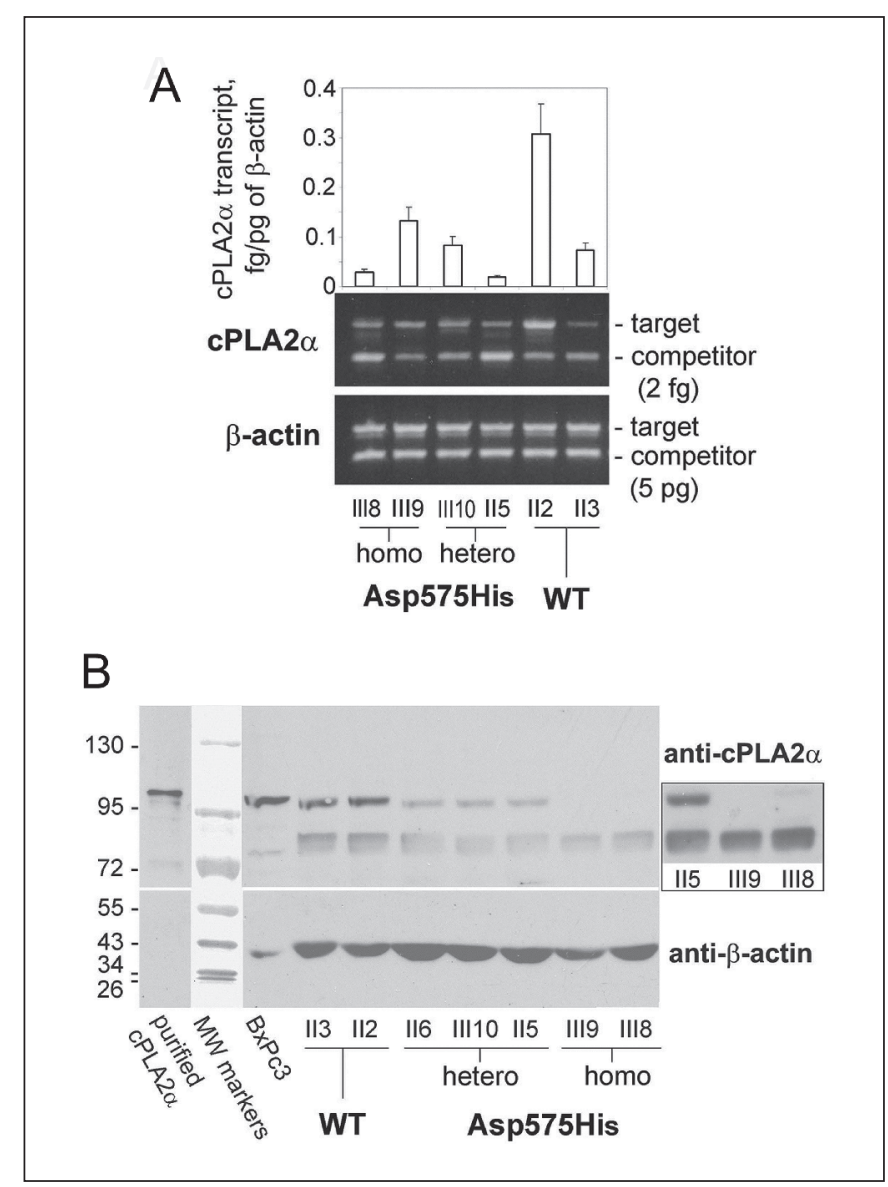

Figure 3: Competitive RT-PCR (A) and Western blotting (B) of cPLA2a. A) Concentrations of target and competitor are indicated besides the gels, and the bar graphs show quantification of the density of the CPLA2a transcript compared to $\beta$-actin. Genotypes are indicated below the gels. B) In the top segment, the membrane was treated with anti-CPLA2a antibodies, in the bottom one with anti- $\beta$ actin antibodies. Samples II2 and II3 are wild-type, samples II6, III10 and II5 are heterozygous and samples III9 and III8 are homozygous for the Asp575His variant (see Figure 2). Purified recombinant and $\mathrm{BXPC} 3$ cell line-derived CPLA2a are shown as reference. In the inset, samples II5, III9 and III8 are shown after prolonged exposure. significant differences associated with genotypes, suggesting that the $1723 \mathrm{G}>\mathrm{C}$ mutation does not affect the transcription of cPLA2a mRNA ( Figure 3A). Conversely, cPLA2a was undetectable by Western Blotting in platelet lysates of the proband and his twin sister, while it was expressed to a lesser extent in platelets from heterozygous subjects, compared to normal subjects ( $\square$ Figure $3 \mathrm{~B}$ ).

These results suggest that $1723 \mathrm{G}>\mathrm{C}$ variant of cPLA2a detected in the kindred can be transcribed, but is not efficiently translated, or is translated into an unstable protein. As a consequence, carriers of the mutation have a deficiency of cPLA2a, which is severe in homozygotes and mild in heterozygotes.

\section{Discussion}

In this report we describe an inherited syndrome, which is characterised by life-long haemorrhagic diathesis associated with platelet dysfunction, and very early-onset GI ulcers. The proband and his twin sister developed duodenal ulcers at 24 months of age and experienced abnormal spontaneous muco-cutaneous bleeding (epistaxis, gum bleeding, hematuria, menorrhagia) and abnormal postsurgical bleeding of moderate severity, which caused the development of iron deficiency anaemia in the twin sister. All these clinical manifestations are associated with severe deficiency of cPLA2a , due to homozygous $1723 \mathrm{G}>\mathrm{C}$ transition in PLA2G4A gene, which changes the codon for Asp575 to His. The proband also displayed renal insufficiency of mild severity.

cPLA2a cleaves AA from membrane phospholipids, making it the rate limiting enzyme for the synthesis of eicosanoids, which are involved in the control of a wide range of physiological processes (1). It is therefore biologically plausible that severe cPLA2a may associate with multiple clinical manifestations.

The bleeding diathesis of our two patients with severe cPLA2a deficiency is associated with defective platelet function, caused by the inability of platelets to form $\mathrm{TxA}_{2}$ from endogenous $\mathrm{AA}$, which is a potent platelet agonist that amplifies the platelet aggregatory and secretory responses stimulated by other platelet agonists (4). The importance of $\mathrm{TxA}_{2}$ in platelet function is demonstrated by the experimental evidence that aspirin, which inhibits the platelet production of $\mathrm{TxA}_{2}$ by irreversibly acetylating the enzyme cyclooxygenase-1 (COX-1), reduces the risk of thrombosis and increases the incidence of bleeding (6). It must be noted, however, that, at variance with the moderate/severe bleeding diathesis displayed by our patients with severe cPLA2a deficiency, the bleeding risk that is associated with aspirin treatment is rather mild. Major bleeding complications of chronic aspirin treatment affect approximately $1-2 / 1,000$ treated patients every year, and are mostly confined to the GI tract (6). The incidence of non-GI bleeding is much more rare (23). The clinical picture of patients on chronic aspirin treatment resembles that of a patient with inherited cPLA2a variants, associated with partial reduction of enzyme activity, due to compound heterozygosity, who was described by Adler et al. $(24,25)$. Though the proband and his family members were affected by small intestinal ulcers, which caused GI bleeding, they did not have a systemic bleeding diathesis (24). Their serum 
$\mathrm{TxB}_{2}$ levels were about one order of magnitude higher than those we detected in the two homozygous carriers of the Asp575His variant, and similar to those of patients on chronic aspirin treatment (24). The degree of impairment of platelet $\mathrm{TxA}_{2}$ production in two recently described patients with cryptogenetic multifocal stenosing enteritis associated with cPLA2a deficiency and no generalised bleeding also appears to be comparable to that induced by aspirin (26). The absence of pathological systemic, extra-GI bleeding in these previously reported patients with inherited cPLA2a is at variance with the moderate/severe bleeding diathesis displayed by our patients with severe cPLA2a deficiency. Two possible causes for their bleeding diathesis are considered.

- The proband and his sister also had very mildly reduced levels of factor XI, which might have synergised with the impaired platelet $\mathrm{TxA}_{2}$ production in increasing the bleeding risk (27). It must be noted, however, that the administration of aspirin to patients with mild factor XI deficiency did not prolong their bleeding time (28), which, in contrast, was very prolonged in our proband. In addition, the mother of our patients also had factor XI deficiency of greater severity ( $42 \%$, vs $64 \%$ and $62 \%$ ), but never experienced pathological bleeding, not even on occasions of ingestion of aspirin.

- The patients reported here also had profoundly low levels of serum $\mathrm{TxB}_{2}(0.17$ and $0.11 \mathrm{ng} / \mathrm{ml})$, suggesting the hypothesis that this great magnitude of suppression of $\mathrm{TxA}_{2}$ contributes to their bleeding diathesis. Despite the clear difference in the bleeding risk, there are no major differences in in vitro platelet function tests between our two patients, the patient described by Adler et al. and aspirin-treated subjects, reflecting the inability of in vitro platelet function tests to predict the risk of bleeding. In vivo, more than one platelet agonist stimulates the platelets at sites of vascular injury. Among these, epinephrine has been demonstrated to synergise with the small concentrations of $\mathrm{TxA}_{2}$ that are produced by platelets of patients treated with aspirin, thereby increasing their ability to form platelet aggregates $(29,30)$. Thus, it is likely that this synergism may not take place when extremely low levels of $\mathrm{TxA}_{2}$ are generated by platelets, as in our two patients (30).

The very early onset of duodenal ulcers in our patients is likely due to the severe deficiency of eicosanoids that protect the GI mucosa. To the best of our knowledge, the occurrence of GI ulcers very early in life is extremely rare. The study of other family members allowed us to identify a clinical gradient of severity in ulcer presentation from wild-type to heterozygous to homozygous individuals: in wild types, gastro-duodenal ulcer occurred at older age $(>60$ years), while in heterozygotes it occurred before 40 years of age, which is rather uncommon in the general population. It is interesting to note that Helicobacter pylori infection was a frequent cofactor of ulcer occurrence in heterozygotes. It is tempting to speculate that carriers of a cPLA2a variant are predisposed to gastro-duodenal ulcer development that is precipitated sooner than in noncarriers, especially when an additional risk factor, such as for example Helicobacter pylori infection, is present. The previously described patients with impaired cPLA2a function had recurrent ulcers of the ileum $(24,26)$; the reason for the selectivity for this GI tract, as opposed to the duodenum is unclear.

Chronic exposure to NSAIDS, which interfere with the generation of prostaglandins by cyclooxygenases, is one of the leading causes of renal functional impairment and chronic renal insufficiency (31). In experimental models, prostaglandins were shown to have complex effects on the blood flow in the kidney $(32,33)$. In our proband, a mild renal insufficiency is present, though it is not observed in his twin sister. It is therefore difficult to draw any certain conclusion regarding the effect of the cPLA2a variant on prostaglandin availability and distribution in the kidney. It is probably safe to suggest that if there is any effect, it is influenced by additional risk factors for kidney disease.

In conclusion, deficiency of cPLA2a may have different clinical presentations, depending on its severity and accompanying predisposing factors. GImucosal protection appears to be very sensitive to even partially reduced levels of prostaglandins. In contrast, primary haemostasis appears to be significantly impaired only in cases of severe defects of eicosanoid production..

\section{Acknowledgements}

We are grateful to Professor Angelo Sala and his collaborators (Dipartimento di Scienze Farmacologiche e Biomolecolari, Università

\section{What is known about this topic?}

- Arachidonic acid, when cleaved from phospholipids by cytosolic phospholipase A2 alpha (CPLA2a), generates gastro-protective, pro-haemostatic, pro-inflammatory, and vasoactive eicosanoids.

- Two cases of CPLA2a deficiency were previously described, with early-onset gastro-intestinal (GI) ulcers, decreased platelet function, but no generalized bleeding diathesis.

\section{What does this paper add?}

- Two patients with CPLA2a deficiency are described, who, in addition to early-onset GI ulcers, also display a generalised, moderate/ severe bleeding diathesis.

- Patients were homozygous for a previously undescribed $1723 \mathrm{G}>\mathrm{C}$ transition in PLA2G4A gene, which changed the codon for Asp575 to His. The Asp575His variant is normally transcribed, but not translated or translated into an unstable protein

- The production of thromboxane A2 by platelets of the patients was extremely low, at least one order of magnitude lower than that observed in previously described patients with CPLA2a deficiency and in individuals on chronic treatment with aspirin,

- The very low levels of platelet thromboxane A2 production, perhaps in combination with the very mild deficiency of coagulation factor XI (about $60 \%$ ) that was detected in the patients, likely account for their bleeding diathesis.

- The study of many members of the large family of our patients revealed that also partial CPLA2a deficiency is associated with increased risk to develop GI ulcers at relative young age, but not with abnormal platelet function or bleeding diathesis. 
degli Studi di Milano, Milan, Italy) for measuring the levels of 12-HETE in serum samples from our patients, and to Dr Gessica Fontana for her help in designing the sequences of the DNA primers. We are grateful to Professor John A. Oates (Departments of Medicine and Pharmacology, Division of Clinical Pharmacology, Vanderbilt University Medical Center, Nashville, Tennessee, USA) for critically reviewing our manuscript.

\section{Conflicts of interest}

None declared.

\section{References}

1. Harizi H, Corcuff JB, Gualde N. Arachidonic-acid-derived eicosanoids: roles in biology and immunopathology. Trends Mol Med 2008; 14: 461-469.

2. Niknami M, Patel M, Witting PK, et al. Molecules in focus: cytosolic phospholipase A2-alpha. Int J Biochem Cell Biol 2009; 41: 994-997.

3. Dennis EA, Cao J, Hsu YH, et al. Phospholipase A2 enzymes: physical structure, biological function, disease implication, chemical inhibition, and therapeutic intervention. Chem Rev 2011; 111: 6130-6185.

4. Mustard JF, Kinlough-Rathbone RL, Packham MA. Prostaglandins and platelets. Annu Rev Med 1980; 31: 89-96.

5. Podda G, Femia EA, Pugliano M, et al. Congenital defects of platelet function. Platelets 2012; 23: 552-563.

6. Patrono C, García Rodríguez LA, Landolfi R, et al. Low-dose aspirin for the prevention of atherothrombosis. N Engl J Med 2005; 353: 2373-2383.

7. Atay S, Tarnawski AS, Dubois A. Eicosanoids and the stomach. Prostaglandins Other Lipid Mediat 2000; 61: 105-124.

8. Tarnawski A, Erickson RA. Sucralfate, 24 years later: current concepts of its protective and therapeutic actions. Europ J Gastroenterol Hepatol 1991; 3: 795-810.

9. Redfern JS, Feldman M. Role of endogenous prostaglandins in preventing gastrointestinal ulceration: induction of ulcers by antibodies to prostaglandins. Gastroenterology 1989; 96: 596-605.

10. Rodeghiero F, Tosetto A, Abshire T, et al. ISTH/SSC joint VWF and Perinatal/ Pediatric Haemostasis Subcommittees Working Group. ISTH/SSC bleeding assessment tool: a standardized questionnaire and a proposal for a new bleeding score for inherited bleeding disorders. J Thromb Haemost 2010; 8: 2063-2065.

11. Cattaneo M. Light transmission aggregometry and ATP release for the diagnostic assessment of platelet function. Semin Thromb Haemost 2009; 35: $158-167$

12. Femia EA, Pugliano M, Podda G, et al. Comparison of different procedures to prepare platelet-rich plasma for studies of platelet aggregation by light transmission aggregometry. Platelets 2012; 23: 7-10.

13. Cattaneo M, Cerletti C, Harrison P, et al. Recommendations for the Standardisation of Light Transmission Aggregometry: A Consensus of the Working Party from the Platelet Physiology Subcommittee of SSC/ISTH. J Thromb Haemost 2013; 11: 1183-1189.

14. Cattaneo M, Lecchi A, Zighetti ML, et al. Platelet aggregation studies: autologous platelet-poor plasma inhibits platelet aggregation when added to plateletrich plasma to normalize platelet count. Haematologica 2007; 92: 694-697.
15. Zarini S, Gijón MA, Ransome AE, et al. Transcellular biosynthesis of cysteinyl leukotrienes In Vivo during mouse peritoneal inflammation. Proc Natl Acad Sci USA 2009, 106: 8296-8301.

16. Ciabattoni G, Maclouf J, Catella F, et al. Radioimmunoassay of 11-dehydrothromboxane B2 in human plasma and urine. Biochim Biophys Acta 1987; 918: 293-297.

17. Mare L, Trinchera M. Comparative analysis of retroviral and native promoters driving expression of betal,3-galactosyltransferase beta3Gal-T5 in human and mouse tissues. J Biol Chem 2007; 282: 49-57.

18. Caretti A, Sirchia SM, Tabano S, et al. DNA methylation and histone modifications modulate the $\beta 1,3$ galactosyltransferase $\beta 3 \mathrm{Gal}-\mathrm{T} 5$ native promoter in cancer cells. Int J Biochem Cell Biol 2012; 44: 84-90.

19. Gilliland G, Perrin S, Blanchard K, et al. Analysis of cytokine mRNA and DNA: detection and quantitation by competitive polymerase chain reaction. Proc Natl Acad Sci USA 1990; 87: 2725-2729.

20. Mannucci PM, Vicente V, Vianello L, et al. Controlled trial of desmopressin in liver cirrhosis and other conditions associated with a prolonged bleeding time. Blood 1986; 67: 1148-1153.

21. Podda GM, Bucciarelli P, Lussana F, et al. Usefulness of PFA-100 testing in the diagnostic screening of patients with suspected abnormalities of haemostasis: comparison with the bleeding time. J Thromb Haemost 2007; 5: 2393-2398.

22. Dolan-O'Keefe M, Chow V Monnier J, et al. Transcriptional regulation and structural organisation of the human cytosolic phospholipase A2 gene. Am J Physiol Lung Cell Mol Physiol 2000; 278: L649-L657.

23. Patrono C, Baigent C. Low-dose aspirin, coxibs, and other NSAIDS: a clinical mosaic emerges. Mol Interv 2009; 9: 31-39.

24. Adler DH, Cogan JD, Phillips JA 3rd, et al. Inherited human cPLA(2alpha) deficiency is associated with impaired eicosanoid biosynthesis, small intestinal ulceration, and platelet dysfunction. J Clin Invest 2008; 118: 2121-2131.

25. Reed KA, Tucker DE, Aloulou A, et al. Functional characterisation of mutations in inherited human $\mathrm{CPLA}_{2}$ deficiency. Biochemistry 2011; 50: 1731-1738.

26. Brooke MA, Longhurst HJ, Plagnol V, et al. Cryptogenic multifocal ulcerating stenosing enteritis associated with homozygous deletion mutations in cytosolic phospholipase A2- $\alpha$. Gut 2014; 63: 96-104.

27. Kitchens CS. Factor XI: A review of its biochemistry and deficiency. Sem Thromb Haemost 1991; 17: 55-72.

28. Kaneshiro MM, Mielke CH Jr, Kasper CK, et al. Bleeding time after aspirin in disorders of intrinsic clotting. N Engl J Med 1969; 281: 1039-1042.

29. Rao GH, Johnson GJ, White JG. Influence of epinephrine on the aggregation response of aspirin-treated platelets. Prostaglandins Med 1980; 5: 45-58.

30. Moscardó A, Santos MT, Fuset MP, et al. Residual cyclooxygenase-1 activity and epinephrine reduce the antiplatelet effect of aspirin in patients with acute myocardial infarction. Thromb Haemost 2011; 105: 663-669.

31. John R, Herzenberg AM. Renal toxicity of therapeutic drugs. J Clin Pathol 2009; 62: 505-515.

32. Hao CM, Breyer MD. Physiological regulation of prostaglandins in the kidney. Annu Rev Physiol 2008; 70: 357-377.

33. Downey P, Sapirstein A, O'Leary E, et al. Renal concentrating defect in mice lacking group IV cytosolic phospholipase A(2). Am J Physiol Renal Physiol 2001; 280: F607-618. 